\title{
The Characteristics of Production Process and the Designning of Automatic Blending System of the Traditional Chinese Spirit
}

\author{
Jianwu Yu ${ }^{1}$, Fangxing $\mathrm{Wu}^{2}{ }^{2}$, , Hao Wang ${ }^{2}$, Hongbo $\mathrm{Li}^{2}$, Xiaoyue Zhang ${ }^{2}$, Yonlin Huang ${ }^{2}$ \\ ${ }^{1}$ Zhejiang Zhonghe Industry Co., Ltd, Hangzhou, P.R. China \\ ${ }^{2}$ Zhejiang SUPCON Co., Ltd, Hangzhou, P.R. China \\ Email address: \\ wufangxing@supcon.com (Fangxing Wu) \\ ${ }^{*}$ Corresponding author \\ To cite this article: \\ Jianwu Yu, Fangxing Wu, Hao Wang, Hongbo Li, Xiaoyue Zhang, Yonlin Huang. The Characteristics of Production Process and the \\ Designning of Automatic Blending System of the Traditional Chinese Spirit. Advances in Applied Sciences. Vol. 5, No. 3, 2020 , pp. 64-69. \\ doi: 10.11648/j.aas.20200503.12
}

Received: June 7, 2020; Accepted: July 6, 2020; Published: August 5, 2020

\begin{abstract}
The Production process of the traditional Chinese spirit is substantially different from beer brewing. Traditional Chinese spirit is produced with sorghum as main raw materials, and mold-based koji as the glycosylation and fermentation agent. The intense-flavor type spirit is an exotic flower in traditional Chinese spirit. It's brewing is an extremely complicated process named continuous slag fermentation. It is characterized by simultaneous steaming and distillation of the mixture of fermented grains and fresh raw materials, and repeated glycosylation and fermentation of the steamed feedstock in solid state in the mud pit. Due to the complexity of the fermentation micro flora and their metabolites, the flavor compositions in the spirit produced from different batches are large different. The proportion of various chemicals in the spirit is large different. This necessitates the development and application of automatic blending system which can improve the blending efficiency and consistency of product quality of the traditional spirit. The automatic control system of brewing and blending is convenient for the production optimization analysis of technologists and provides a strong guarantee for exploring the optimal production parameters. Today most large scale distilleries begin to use the automatic blending control system to improve production efficiency and production capacity.
\end{abstract}

Keywords: Spirit, Koji, Glycosylation, Fermentation, Automatic Blending

\section{Introduction}

Living in 7,000 BC, the Neolithic ancestors of the Chinese have begun to ferment alcoholic drink. China is the birthplace of koji. The use of the mold to ferment alcoholic drink is one of the original winemaking techniques in the world. The fermentation starter is based on certain types of medium which facilitate growth of mildew [1]. Koji is Japanese pronunciation of the fermentation starter which is moldy wheat. Chinese distilled spirit has 1792 years of recorded history beginning in the Eastern Han Dynasty. The fermentation characteristics of the Chinese traditional spirit are repeated and solid state fermentation. The feedstock is glycosylated and fermented simultaneously. The process is amylo process and fermentation.
Koji is actually evolved from the moldy grain. In primitive societies, the moldy or sprouted grain due to improper preservation was found able to be fermented into alcoholic drink. Therefore, these moldy, or sprouted grain became the original koji, it is fermentation feedstock also.

Based on their flavors, Chinese distilled spirits can be classified into 5 types: the soy source flavor, the intense-flavor, the light -flavor, the rice-flavor and the other flavors.

Spirit different in flavor types adopt different koji and the fermentation process is different also. The fermentation starter of intense-flavor spirit is called "big size koji”, while the fermentation starter of light -flavor spirit is "small size koji”. It is not only different in dimension, but also in microbial species involved. The preparation of koji adopts 
solid state fermentation.

\section{The Production Method of Big Size Koji}

It is necessary to introduce the koji production method before expound fermentation characteristics.

The preparation of koji adopts solid state fermentation. The production principle of the big size koji is to maximize the growth of mold and other microbes which are suitable for fermentation.

The raw materials of the big size koji are mainly wheat, barley, plus a certain percentage of peas. The feedstock is added with right amount of water. After kneading the Koji feedstock is then stampeded into a larger size block by specialized workers. So this kind of koji is called big size koji. Stampede is a difficult and interesting work. The workers are strictly specified in their works. The stampede and De-moulding method is shown in Figure 1 and Figure 2. Some people devoted to the measurement of feedstock and water, some specialized to mixing of feedstock, some specialized in putting raw material into the wooden mould and trampled. After de-moulding, the blocks are moved to the breeding room.

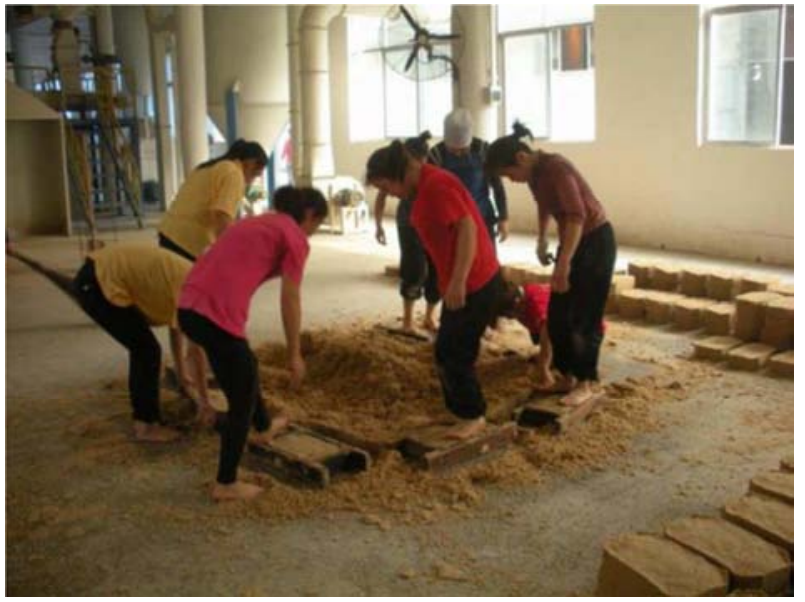

Figure 1. Stampede.

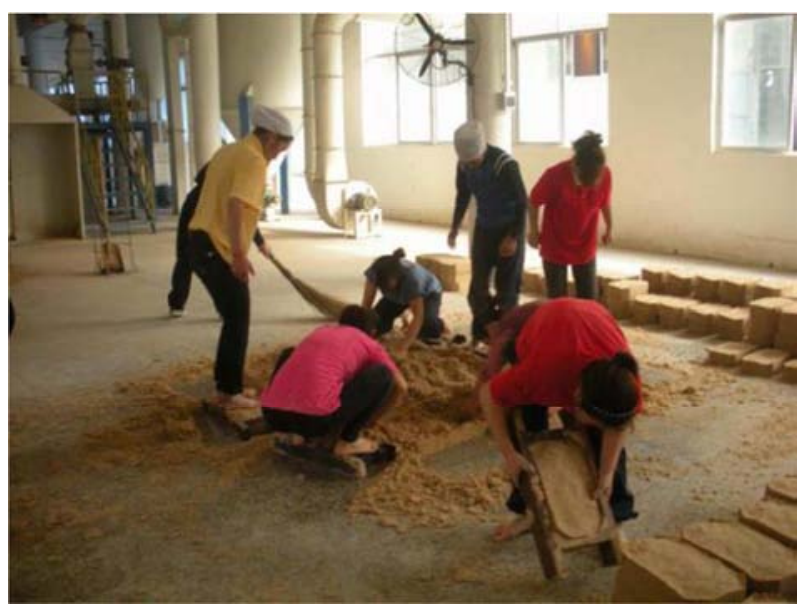

Figure 2. De-moulding.

\subsection{Incubation Temperature}

The microbial thallus is naturally inoculated through a variety of sources [6]. In the closed breeding room, microbes begin to grow, and release heat, which accelerates the evaporation of water. The breeding room is shown in Figure 3. During inoculation the temperature and humidity are rising. The fermentation temperature of the big size koji could be up to $50-60^{\circ} \mathrm{C}$.

Higher fermentation temperature of koji is one of important reasons that the spirit produced with big size koji can get intensified aroma.

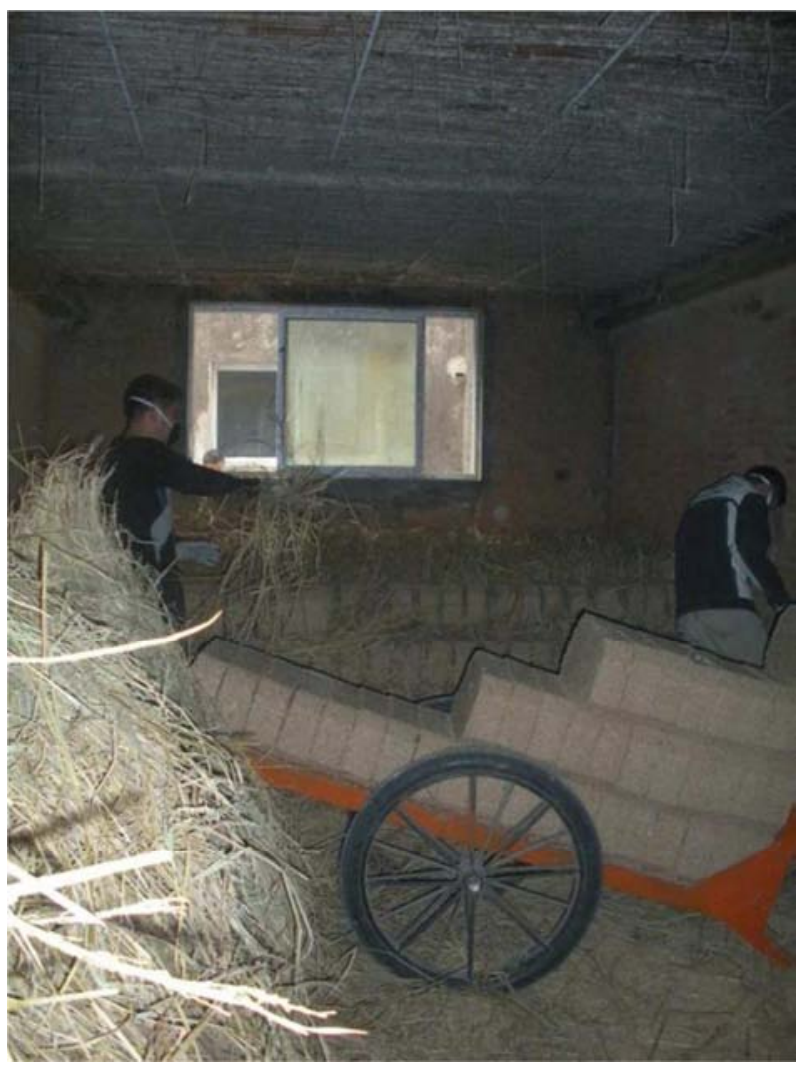

Figure 3. Placement of the koji block in breeding room.

\subsection{Koji Microbial Species and Functional}

The microorganisms in koji are mainly four categories: mold, bacteria, yeast and small number of actinomycetes.

The main mold in Koji include: Aspergillus (Aspergillus), Rhizopus (Rhizopus), Red Aspergillus (Monascus), Mucor (Mucor), Penicillium (Penicillium), Pear head spp (Absidia).

Rhizopus consists Rhizopus nigricans, Rhizopus oryzae and China Rhizopus arrhizus mold. Rhizopus oryzae plays the primary role in koji. It has a strong glycosylation as well as fermentation capacities.

Bacteria play an important role in the fermentation process to produce flavoring substances [11]. There are mainly three types of bacteria in koji: lactic acid bacteria (latobacillus), acetate genus (Acetobacter) and bacillus subtilis (Bacillus subtills).

The yeast in Koji include: Sacharomyces cerevisiae, pichia 
and Candida. Sacharomyces cerevisiae is the predominant yeast in koji. The quantity of cerevisiae is not much compared to other yeast, but it plays a decisive role in the quality of koji and spirit.

In fact koji production is a process which needs to obtain comprehensive balance of microbial flora and their corresponding metabolites [10].

\section{Traditional Production Technology}

\subsection{Fermentation Vessel}

The diversity of the fermentation containers is also one of the main factors to contribute to the difference in spirit flavor There are mainly two types of traditional fermentation container: pottery jars and cellar. Pottery jars are sub-divided into the type buried below ground and the type placed indoor.

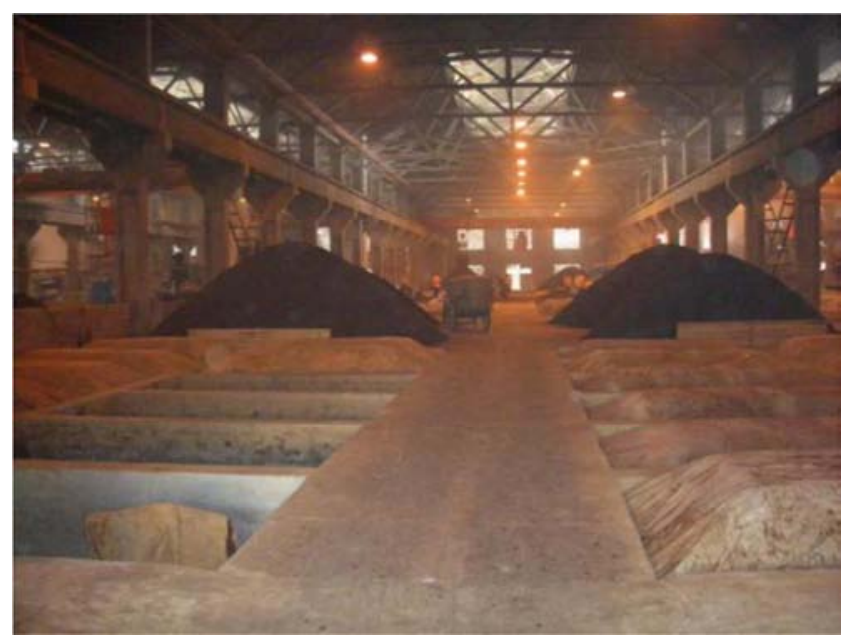

Figure 4. Mud pit, the fermentation vessel for intense-flavor spirit.

The cellar is a special fermentation vessel. It is a big mud pit built by excavation into the ground, in which the feedstock is accumulated for fermentation naturally. The fermentation vessel is shown in Figure 4.

\subsection{The Raw Material for Intense-flavor Spirit Fermentation}

The starchy material for intense-flavor spirit include: sorghum as the main feedstock, barley, wheat, and accompanied by a certain percentage of pea. The brewing process is extremely complicated. The major processes include distillation and steaming of the mixture composed of distiller's grains, rice bran, and feedstock simultaneously. The main advantage to the continued slag method is the improved utilization of feedstock after several times fermentation. The repeated fermentation is also conducive to the accumulation of flavor compounds. The distillation and the steaming of new feedstock at the same time would bring some of the aroma components in fresh ingredients into the spirit. Addition of the rice bran as filler helped to maintain fermented grains loose and thus facilitating steam circulation. During the fermentation, the rice bran also played the role of diluting starch concentration, buffering the acidity, absorption of alcohol, and holding the water generated in the fermentation process. The Components of the water generated in fermentation process include soluble starch, yeast autolysis matters, reducing sugar, tannin, pigment and other soluble materials in feedstock.

The flavors of spirits collected from different point of distillation process were significantly different. So the original spirits are evaluated, graded, and then put into storage between six months to three years for maturation.

\subsection{The Storage and Ripening of the Spirit}

The taste of newly distilled spirit has a spicy sense and other unpleasant odors because of the presence of some sulfide. The new spirit must be stored in pottery jars for maturation. After maturation, the composition of flavor substances is improved and the taste of the spirit becomes mellow and smooth. Finely the matured spirits are blended to yield varied final products each with distinct and unified flavor. The storage and ripening container is shown in Figure 5.

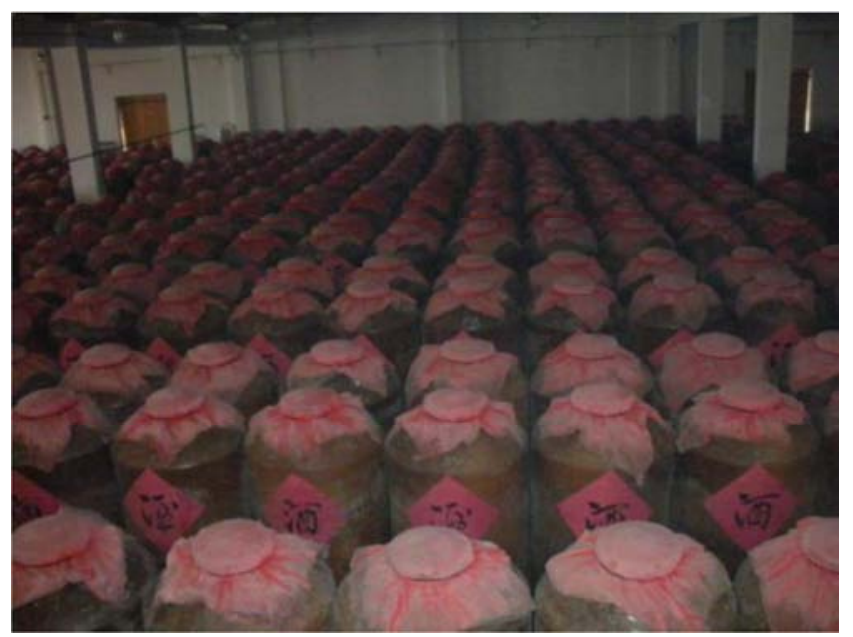

Figure 5. Storage in pottery jars.

\subsection{Blending Technology}

The koji used in fermentation is produced with natural inoculation. The sites for fermentation production are also open. Therefore the qualities of the original spirits from different batches are extremely inconsistent. There are hundreds kinds of trace components in the produced original spirit. They are mainly affected by the characteristics of raw material, fermentation process and distillation. The content of a considerable portion of them is extremely low, the sum amount of them is hardly more than $2 \%$, but their roles are significant. For certain components, their balances rather than their absolute contents are the key to the style and quality of a spirit.

So it is difficult to maintain quality stability and unique style of the products without blending. Blending and flavoring technology is thus a very important part of the spirit 
production techniques; it has played a very significant role to stabilize the spirit quality, to increase the proportion of high-quality spirit.

Flavoring is the key to master the style and adjust the quality of the spirit. This is done by controlled addition of some aromatic substances into the base spirit. It will cause changes in spirit, so as to achieve balance and formation of a fixed style.

Traditionally, blending was undertaken by an experienced brew master according to his sensory evaluation. The judgment of the blending amount is according with the ruler installed on the tank. So the blending ratio is extreme inaccuracy and the success rate is very low. This may result fluctuation of quality of a spirit. Manual blending is also labor-intensive and low efficiency.

Automatic blending, developed after chemical and instrumental analysis of compositions in spirits combined with pilot test, offered accurate measurement of the blending ratio of each raw spirits and the amount of the added flavoring liquor. It can compensate defects of the semi-finished products caused by objective factors to improve the quality of the final spirit product. This enables the products to achieve uniform standards to ensure long-term stability and improve the quality of the spirit [3].

Today most large scale distilleries begin to use the automatic blending control system to improve production efficiency and production capacity [5].

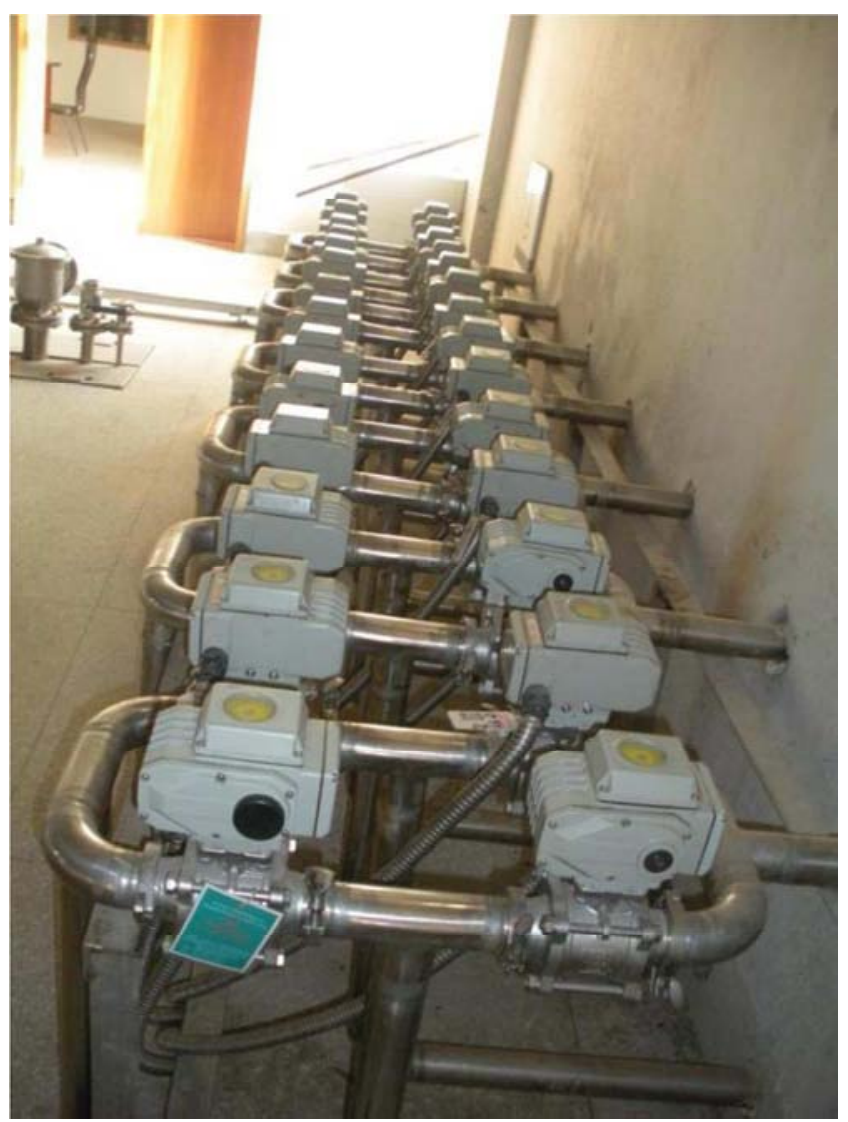

Figure 6. Conversion valve group.

\section{The Design of Blending Pipelne and Automatic Control System}

\subsection{Flexible Pipeline Design for the Blending Process}

The ideas of our process pipeline design are below:

First of all, the tankfarm be constituted to relatively independent blending production unit according to the process requirements. So that it can connect with each functioned tank on tankfarm.

The second, the pipeline can cross regional arbitrary interworking. It meets the requirement of flexible scheduling in the actual production in special blending requirements need.

The third, The precision of the spirit blending had be enhanced by the use of filtration line, blending line and ionized water line to realize the water leading and pushing.

The Fourth, We define the pipeline selection and pump use principle to ensure the realization of complex blending process automatic control. The conversion valve group is shown in Figure 6.

\subsection{Overall Scheme Design of the Blending Automatic Control System}

The important jobs are not only process piping design but also automatic control strategies to improve the efficiency. In order to improve production efficiency and reducing waste, it is necessary that the control strategy designed takes advantage of the flexibility of the process pipeline [2].

The blending system realize that the spirit can be automatic transfer and blending between each tank flexibly because the pipeline possesses commonality and flexibility. The precision of the spirit blending had be enhanced by the use of filtration line, blending line and soft water line to realize the water leading and pushing.

The project adopted the ideas of a decentralized control and centralized dispatching management [8]. For each region are designed respectively corresponding to the control room for local control and decentralized control system can realize data sharing of the whole project system data and upper management network by OPC technology [9]. The overall configuration of blending control system is shown in Figure 7.

The Blending automatic control system can automatically blend various raw materials according to the formula. Real-time communication is through Ethernet e.g. bus for connecting on-site $\mathrm{I} / \mathrm{O}$ station and operation/engineer station.

The operation/engineer station is used for the formula filling and monitoring of the process. The state and malfunction alarm of level transmitters, pumps and electric ball valve are indicated in the interface. When the valve abnormal opening or tank level abnormal changes appeared, the system automatically stops running. Until the operator confirm the troubleshooting be eliminated, the system will continue to run. 


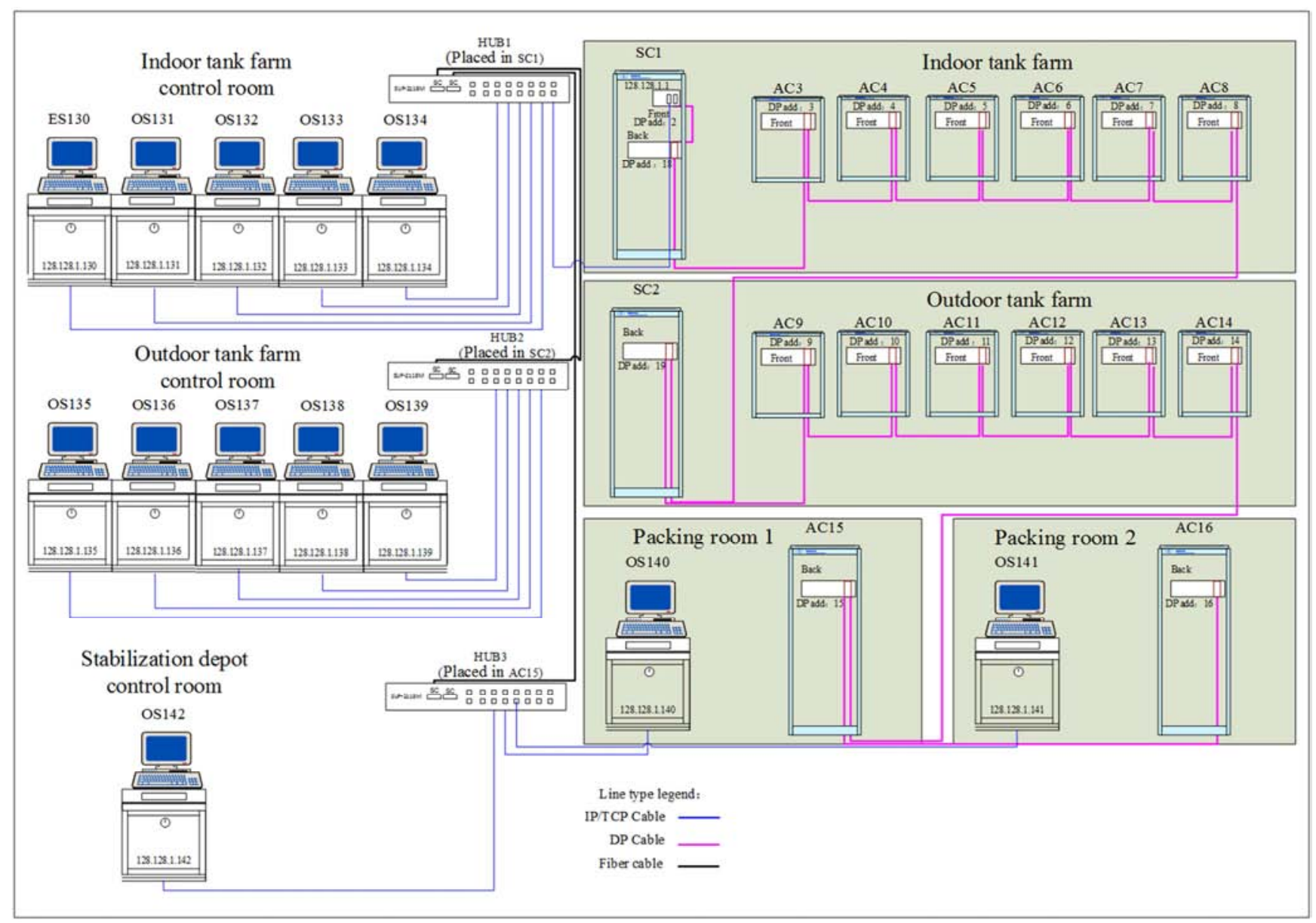

Figure 7. Overall configuration diagram of the blending system.

\section{Conclusions}

Due to the numerous types of flavor substances existing in Chinese spirits and the complexity of the blending process, the efficiency of manual bending is lower. The automatic blending control system specifically developed in accordance with unique requirements of a given process, is able to automatically precisely control the amounts of each of the original spirits and flavoring liquors added during the real blending process. The blending automatic control helps to improve production efficiency, capacity and quality [7].

\section{Acknowledgements}

We got the strong guidance of the professor Tiejin Ying of Institute of Food Science, Zhejiang University during the paper writing. I would like to express my sincere thanks.

\section{References}

[1] Joseph Needham. Science And Civilisation In China Vol 6 Biology and biological technology Part 5 Fermentations and food sience, Cambridge University Press, 2000.
[2] Yang, Tuanyuan. Wu, Fangxing. The design and application of the technical pipeline and automatic blending control system for liquor. Liquor Science. 196 (9), 2010, P53-55 (in Chinese).

[3] Lian, Ruifeng. Computer simulation of Liquor blending and seasoning. [J] Metallurgical Automation, 2006 (sul), P48-51 (in Chinese).

[4] Xie, Wenyong. Automatic control system in the production of liquor. [J] Liquor-making Science \& technology,. 159 (9), 2007, P53-58 (in Chinese).

[5] Yang, Zhiyong. The application of automatic control system in the "Jing" wine production process [J] Liquor-making Science \& technology, 147 (9), 2006, P60-62 (in Chinese).

[6] Shen, Yong xiang. Wu, Fangxing. The new brewing process of the Maple forest brewery and its significance to the automation development of traditional liquor industry. [J] Liquor-making Science \& technology. Vol • 196, No. 10, 2010 P53-55 (in Chinese).

[7] Zhao, deyi. Han, bin. Wu, fanxing. et al. The liquor production automation system of Shandong Jingzhi Liquor Co., Ltd. [J] Liquor Science, Vol·240, No. 6, 2014 P91-94 (in Chinese).

[8] Jiao, erman. Wu, fanxing. et al. The ponder on composite automation of the whole plant for the traditional Chinese spirit enterprises. [J] Liquor Science, Vol·227, No. 5, 2013 P117-121 (in Chinese). 
[9] Gao, Jiakun. Tang, Youhong. Cui, Yujun. Li, Anjun. Gao, Lijia. Research on Automatic Transformation of Liquor Solid Fermentation. [J] Liquor Making, Vol. 46, No. 1, Jan. 2019, P78-P80 (in Chinese).

[10] Luo, Jing, Zhu, Shuilan, Wang, Li, He Jialin, Ouyang, Linghua. Zou, Jinying. Research progress on the composition of brewing microorganisms and flavor substances in strong-flavorBaijiu. [J] China Brewing, 2020, Vol. 39, No. 4, P1-5 (in Chinese).

[11] Sun, Lilin. Li, Lilang. Hu, Ping. Tian, Ya. Ma, Yingyao. Yuan, Zaishun. Analysis of bacterial diversity during the fourth round of sauce-flavor Baijiu fermentation. [J] China Brewing, 2020, Vol. 39, No. 5, Serial No. 339. p 35-39 (in Chinese). 